\title{
11. STRONTIUM ISOTOPE GEOCHEMISTRY OF LEG 113 INTERSTITIAL WATERS AND CARBONATES ${ }^{1}$
}

\author{
Per Kristian Egeberg, ${ }^{2}$ P. Craig Smalley, ${ }^{3}$ and Per Aagaard ${ }^{2}$
}

\begin{abstract}
The concentration of dissolved $\mathrm{Sr}$ and the distribution of ${ }^{87} \mathrm{Sr} /{ }^{86} \mathrm{Sr}$ isotope ratios in Leg 113 interstitial waters may be interpreted in terms of mixing of $\mathrm{Sr}$ from four different reservoirs: indigenous seawater, marine carbonate minerals, and basaltic and siliceous detrital material. The input to the pore water from these reservoirs is determined by the reactivity of the reservoir rather than its size. The presence of strontium derived from siliceous detrital material is unequivocally demonstrated in the pore waters of the hemipelagic deposits, and is also significant in the calcareous Maud Rise sediments due to the unusually low degree of carbonate recrystallization. Also, alteration of basic volcanic material is important at several sites.
\end{abstract}

\section{INTRODUCTION}

In marine sediments the bulk of the elements is held in the solid phases while the pore water serves as a conduit between reacting solid phases and their products. Thus, pore waters are very sensitive indicators of incipient diagenetic reactions. Variations in the chemical composition of interstitial waters have proven to be useful for the delineation of elemental budgets (e.g., Manheim, 1976; Gieskes and Lawrence, 1976; Baker, 1986). However, because several different mechanisms may leave essentially the same imprint on the concentration profiles of dissolved constituents, major element chemistry on its own is not always sufficient to fingerprint reactions. For example, the frequently observed depletion of pore water magnesium and concomitant enrichment of calcium (Gieskes, 1973; McDuff, 1981; Baker, 1986) have been attributed to dolomitization (Manheim, 1976), reversed weathering (Wollast, 1974), and to alteration of volcanic matter dispersed in the sediments (Perry et al., 1976) or associated with the volcanic basement (McDuff, 1981; Baker, 1986). Because the amounts of authigenic phases formed are generally too small to be quantified reliably, only in special situations will petrographic examinations be diagnostic.

${ }^{87} \mathrm{Sr} /{ }^{86} \mathrm{Sr}$ isotope ratio determinations of $\mathrm{Sr}$ dissolved in the pore water add an extra dimension to such studies. For example Hawkesworth and Elderfield (1976) were able to identify alteration of volcanically derived material and recrystallization of carbonates as the controlling mechanisms, rather than pore water/basement interactions. Subsequently, several similar studies summarized by Elderfield and Gieskes (1982) have shown that ${ }^{87} \mathrm{Sr} /{ }^{86} \mathrm{Sr}$ isotope ratios are sensitive indicators of diagenetic reactions.

Other applications of pore water/rock strontium isotope geochemical studies include studies of seawater-oceanic crust interactions (Spooner, 1976; Palmer and Elderfield, 1985) and determination of the rate of recrystallization of carbonates in pelagic carbonate deposits (Richter and Depaolo, 1987).

The main aims of the present work are to establish the distribution of ${ }^{87} \mathrm{Sr} /{ }^{86} \mathrm{Sr}$ isotope ratio in pore water and carbonates recovered during Leg 113, and to delineate the controlling mechanisms.

\footnotetext{
${ }^{1}$ Barker, P. F., Kennett, J. P., et al., 1990. Proc. ODP, Sci. Results, 113: College Station, TX (Ocean Drilling Program).

2 Department of Geology, P.O. Box 1047, Blindern, N-0316 Oslo 3, Norway.

3 Institute for Energy Technology, P.O. Box 40, Kjeller, N-2007, Norway.
}

\section{ANALYTICAL METHODS}

The interstitial water were extracted on board using a stainless steel hydraulic press and the procedures described by Manheim and Sayles (1974). The concentration dissolved strontium was determined by atomic absorption spectrophotometry (AAS) after adjusting the samples to $4000 \mathrm{ppm} \mathrm{La}^{3+}$ with $\mathrm{LaCl}_{3}$ (Gieskes, 1973). For ${ }^{87} \mathrm{Sr} /{ }^{86} \mathrm{Sr}$ isotope analysis, $\mathrm{Sr}$ was concentrated from pore water samples using miniature ionexchange columns. The strontium concentrates were analyzed on a Finnigan MAT 261 machine (Institute of Energy Technology) in the static multicollector mode. ${ }^{87} \mathrm{Sr} /{ }^{86} \mathrm{Sr}$ ratios were normalized to $86 \mathrm{Sr} / 88 \mathrm{Sr}=$ 0.1194 . Measurements of the NBS 987 standard during the period of analysis averaged $0.710251(2 \sigma=0.00003)$.

A 1-hr treatment of bulk sediments with cold $0.1 \mathrm{~N} \mathrm{HCl}$ was used to liberate adsorbed strontium, strontium in exchange positions, and strontium held in carbonates. The ${ }^{87} \mathrm{Sr} /{ }^{86} \mathrm{Sr}$ ratio of the leached strontium was subsequently determined as described above. The concentration of calcium, magnesium, and strontium in the acid extract were determined by AAS as described above and back-calculated to percent (Ca) and ppm (Mg and $\mathrm{Sr}$ ) of bulk sample (only for Sites 689 and 690 ).

The $\delta^{18} \mathrm{O}$ values of the pore waters were determined using the $\mathrm{CO}_{2}$ equilibration method. Isotope analyses were performed on a Finnigan MAT 251 mass spectrometer. The results are reproducible to $\pm 0.2 \%$ ( 2 sigma).

\section{SITE DESCRIPTION}

\section{Site 689 (Maud Rise)}

The site is located near the crest of Maud Rise (Fig. 1) at a water depth of $2080 \mathrm{~m}$. Whole round samples for squeezing were taken from cores collected from Hole 689B drilled to 297.3 mbsf, terminated approximately $40 \mathrm{~m}$ above basement. The recovered sediments are pelagic, biogenic in origin, and range in age from Campanian/Maestrichtian to Pleistocene. The sequence has been divided into three lithostratigraphic units based upon compositional differences and diagenetic maturity.

Unit I (0-31.0 mbsf; late Miocene to Pleistocene) consists predominantly of diatom ooze with varying amounts of other biosiliceous components.

Unit II (31.0-149.1 mbsf; late Eocene to late Miocene) consists of a mixture of biosiliceous and calcareous oozes. The calcareous component (mainly nannofossils) increases downward.

Unit III (149.1-297.3 mbsf; Campanian? to late Eocene) consists of nannofossil ooze and chalk.

Zeolites, chert, and altered volcanic ash-bearing layers are minor components. The clay size fraction (Barker, Kennett, et al., 1988) is dominated by smectite and illite down to $110 \mathrm{mbsf}$ and by smectite below 110 mbsf. 


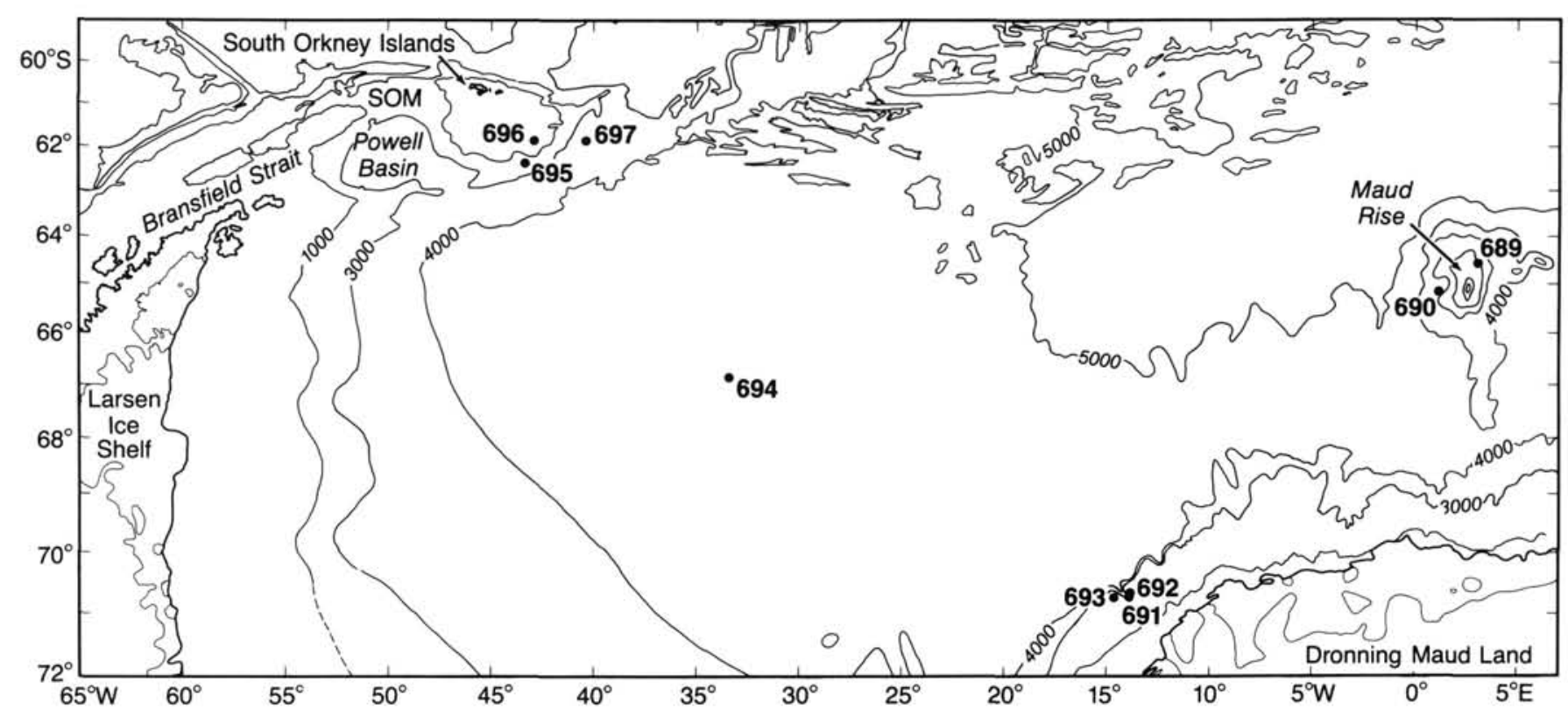

Figure 1. ODP Leg 113 site locations.

\section{Site 690 (Maud Rise)}

This site is situated on the southwest flank of Maud Rise in $2914 \mathrm{~m}$ of water. Whole round samples for squeezing were taken from cores from Hole 690B drilled to $213.4 \mathrm{mbsf}$ and from Hole $690 \mathrm{C}$, terminated in basaltic rock at 321.2 mbsf. The sediments range in age from late Cretaceous to Pleistocene. The sequence is mainly biogenic, but terrigenous and volcanic detritus become a significant component around $140 \mathrm{mbsf}$. Thin, rare, chert layers were recovered. The sequence has been divided into six lithostratigraphic units.

Unit I (0-24.4 mbsf; late Miocene to Pleistocene) consists of $2.1 \mathrm{~m}$ of foraminiferal ooze overlying diatom ooze with varying amounts of other biosiliceous components.

Unit II (24.4-92.9 mbsf; early Oligocene to late Miocene) consists of pure and mixed biogenic siliceous and calcareous oozes.

Unit III (92.9-137.8 mbsf; late Paleocene to early Oligocene) consists of foraminiferal oozes.

Unit IV (137.8-281.1 mbsf; late Maestrichtian to late Paleocene) consists of nannofossil oozes and chalks with a terrigenous component of $10 \%-15 \%$.

Unit V (281.1-317.0 mbsf; late Campanian(?) to late Maestrichtian) consists predominantly of terrigenous material (including volcanic glass) and contains calcareous ooze and chalk.

Unit VI (317.0-321.1 mbsf) consists of $1.71 \mathrm{~m}$ of amygdaloidal pyroxene olivine basalts.

The clay size fraction (Barker, Kennett, et al., 1988) is dominated by smectite except in the upper 90 mbsf where illite is equal to or more common. Chlorite + kaolinite is present down to $213 \mathrm{mbsf}$. Zeolites are present at $230-260 \mathrm{mbsf}$.

\section{Site 693 (Dronning Maud Land margin)}

Site 693 lies on a mid-slope bench of the Weddell Sea margin of East Antarctica in $2359 \mathrm{~m}$ of water. Whole round samples for squeezing were taken from cores from Hole 693A drilled to $474.3 \mathrm{mbsf}$ and from Hole 693B drilled to $403.1 \mathrm{mbsf}$. The seismic records indicate the presence of at least another $1000 \mathrm{~m}$ of sediments below our maximum penetration. The sediments range in age from lower Cretaceous to Pleistocene. The sequence has been divided into seven lithostratigraphic units.
Unit I (0-12.2 mbsf; Pleistocene) consists of foraminiferbearing clayey mud.

Unit II (12.2-31.4 mbsf; late Pliocene to Pleistocene) consists of clayey mud.

Unit III (31.4-325.8 mbsf; late Oligocene to late Pliocene) consists of diatom mud and diatom-bearing silty to clayey mud.

Unit IV (325.8-345.1 mbsf; late Oligocene) consists of alternating diatomaceous mud and ooze and minor muddy nannofossil ooze and nannofossil-bearing clayey mud.

Unit V (345.1-397.8 mbsf; Oligocene) consists predominantly of diatomaceous mud and silt.

Unit VI (397.8-409.0 mbsf; Cretaceous-?Albian to Santonian) consists of radiolarian diatomite.

Unit VII (409.0-483.9 mbsf; early Cretaceous-Albian) consists of terrigenous claystones and mudstones with organic rich beds.

The clay size fraction (Barker, Kennett, et al., 1988) is dominated by illite down to 403.1 mbsf and by smectite from 403.1 mbsf to 483.9 mbsf. Kaolinite and chlorite are present in most samples above 403.1 mbsf. Volcanic glass and opaques are present throughout as minor components.

\section{Site 694 (Weddell abyssal plain)}

Site 694 is situated on the northern part of the Weddell abyssal plain in $4653 \mathrm{~m}$ of water. Whole round samples for squeezing were collected from cores from Hole 694B drilled to 179.2 mbsf and from Hole $694 \mathrm{C}$ terminated at $391.3 \mathrm{mbsf}$. The seismic records indicate the presence of more than $1000 \mathrm{~m}$ of sediment below our total depth. The recovered sediments range in age from middle Miocene to Pleistocene and are mostly terrigenous with a minor biosilicious component. Four lithostratigraphic units were defined.

Unit I (0-21.1 mbsf; early Pliocene to Pleistocene) consists of terrigenous clay and clayey mud with minor silt and diatombearing clayey mud.

Unit II (21.1-111.5 mbsf; early Pliocene?) consists predominantly of well- to moderately-well-sorted lithic and quartz sands.

Unit III (111.5-304.3 mbsf; middle Miocene? to early Pliocene?) consists of hemipelagic sediments and turbidites, graded silt sequences with some diatoms and diatom-bearing silty and clayey muds with interbedded silts and sandy muds. 
Unit IV (304.3-391.3 mbsf; middle Miocene) consists of diatom-bearing and diatomaceous claystones, with silt near the base.

The clay size fraction (Barker, Kennett, et al., 1988) is dominated by illite and chlorite. The proportion of chlorite increases downward while the abundance of kaolinite and smectite generally decrease toward the base of the sequence. Opaques are abundant.

\section{Site 695 (South Orkney microcontinent)}

Site 695 is located on the southeastern edge of the South Orkney microcontinent in $1305 \mathrm{~m}$ of water. Whole round samples for squeezing were sampled from cores recovered from Hole $695 \mathrm{~A}$ drilled to $341.1 \mathrm{mbsf}$. The seismic records indicate the presence of at least another $1500 \mathrm{~m}$ of sediment below. The sediments are biosiliceous hemipelagic deposits and range in age from Miocene/Pliocene to Pleistocene. Three lithostratigraphic units were defined.

Unit I (0-190.0 mbsf; Pleistocene to early Pliocene) consists of diatom-bearing silty and clayey muds, muddy diatom ooze, diatom ooze, and diatom silty muds.

Unit II (190.0-306.9 mbsf; early Pliocene) consists of diatom-bearing silty and clayey muds.

Unit III (306.9-341.1 mbsf; early Pliocene to late Miocene) consists of silty mud with $0 \%-10 \%$ diatoms.

The clay size fraction (Barker, Kennett, et al., 1988) is dominated by illite and chlorite, the proportion of kaolinite decreases downhole, while the relative abundance of smectite varies randomly. Volcanic glass and rock fragments are observed throughout.

\section{Site 696 (South Orkney microcontinent)}

Site 696 is situated on the southeastern margin of the South Orkney microcontinent, under $650 \mathrm{~m}$ of water. Whole round samples for squeezing were taken from cores from Hole 696A drilled to 106.0 mbsf and from Hole 696B terminated at 645.6 mbsf. The seismic records indicate total sediment thickness of at least $1200 \mathrm{~m}$. The sediments are terrigenous, hemipelagic, and pelagic and range in age from middle/upper Eocene to Pleistocene. The sequence has been divided into seven lithostratigraphic units.

Unit I (0-64.2 mbsf; Pleistocene to early late Pliocene) consists primarily of diatomaceous muds and oozes.

Unit II (64.2-124.8 mbsf; early late Pliocene to early Pliocene) consists mainly of diatom-bearing silty and clayey mud.
Unit III (124.8-211.8 mbsf; early Pliocene to late Miocene) consists predominantly of silty and clayey mud, with less important diatom-bearing clayey mud.

Unit IV (211.8-260.1 mbsf; late Miocene) consists of diatom ooze and muddy diatom ooze.

Unit V (260.1-269.7 mbsf; late Miocene) consists of coarsegrained sand.

Unit VI (269.7-529.8 mbsf; late Miocene to middle Miocene) consists of diatom ooze, mud-bearing diatom ooze, diatomite, and mud-bearing diatomite.

Unit VII (529.8-645.6 mbsf; middle? to late Eocene) consists of sandy mudstone, claystone, clayey mudstone, silty mudstone, and barren glauconitic silty mudstone.

The clay size fraction (Barker, Kennett, et al., 1988) is dominated by chlorite and illite down to $500 \mathrm{mbsf}$ and by smectite from 500 mbsf to $645.6 \mathrm{mbsf}$. Volcanic ash is present as layers throughout most of the sequence and is particularly abundant between 269.7 and $529.8 \mathrm{mbsf}$; finely dispersed glass constitutes $4 \%-8 \%$ of the sediment (smear slide estimates).

\section{Site 697 (South Orkney microcontinent-Jane Basin)}

Site 697 is located in Jane Basin under $3484 \mathrm{~m}$ of water. Whole round samples for squeezing were taken from cores from Hole 697A drilled to 20.9 mbsf and from Hole 697B terminated at 322.9 mbsf. The seismic records indicate a total sediment thickness of at least $800 \mathrm{~m}$. The recovered sediments are hemipelagic with a minor biosilicious component, and range in age from Pliocene to Pleistocene. Two lithostratigraphic units were defined.

Unit I (0-293.0 mbsf; Pliocene to Pleistocene) consists of silty mud, diatom-bearing silty mud, clayey mud, and diatombearing clayey mud.

Unit II (293.0-322.9 mbsf; early Pliocene) consists of silty and clayey mud.

The clay size fraction (Barker, Kennett, et al., 1988) is dominated by illite and chlorite; kaolinite and smectite are present at most depths. Volcanic ash is abundant.

\section{RESULTS}

The strontium isotope data are presented in Tables 1-7 and compared to the seawater ${ }^{87} \mathrm{Sr} /{ }^{86} \mathrm{Sr}$-time curve (data from DePaolo and Ingram, 1985; Palmer and Elderfield, 1985; DePaolo, 1986; Koepnick et al., 1985; Hess et al., 1986) on Figures 2-5. The shaded areas represent the spread of data used to draw the time curve. For the calcareous sequences (Sites 689 and 690) we

Table 1. Interstitial water concentration data and concentration of elements in bulk sediment (extracted with $0.1 \mathrm{~N} \mathrm{HCl}$ ), Site 689.

\begin{tabular}{|c|c|c|c|c|c|c|c|c|c|}
\hline $\begin{array}{l}\text { Core, section, } \\
\text { interval }(\mathrm{cm})\end{array}$ & $\begin{array}{l}\text { Depth } \\
\text { (mbsf) }\end{array}$ & $\begin{array}{l}\text { Age } \\
(\mathrm{Ma})\end{array}$ & $\begin{array}{l}\mathrm{Sr}^{2+} \\
(\mu \mathrm{m})\end{array}$ & $\begin{array}{c}{ }^{87} \mathrm{Sr} /{ }^{86} \mathrm{Sr} \\
(\text { I.W })^{\mathrm{a}}\end{array}$ & $\begin{array}{l}\delta^{18} \mathrm{O} \\
(\%)^{\mathrm{b}}\end{array}$ & $\begin{array}{c}{ }^{87} \mathrm{Sr} /{ }^{86} \mathrm{Sr} \\
\text { (A.E.) }{ }^{\mathrm{c}}\end{array}$ & $\underset{(\mathrm{ppt})^{\mathrm{c}}}{\mathrm{Ca}}$ & $\underset{(\mathrm{ppm})^{\mathrm{c}}}{\mathrm{Sr}}$ & $\underset{(\mathrm{ppt})^{\mathrm{c}}}{\mathrm{Mg}}$ \\
\hline \multicolumn{10}{|l|}{ 113-689B- } \\
\hline $1 \mathrm{H}-3,78-85$ & 3.78 & 3.8 & & & & 0.70946 & 0 & 12 & 0.9 \\
\hline $1 \mathrm{H}-3,145-150$ & 4.45 & 4.1 & 101 & 0.70902 & 0.2 & 0.70926 & 2 & 19 & 1.2 \\
\hline $2 \mathrm{H}-2,66-70$ & 7.46 & 4.5 & 104 & 0.70905 & 0.7 & 0.70937 & 1 & 16 & 0.5 \\
\hline $3 \mathrm{H}-4,120-125$ & 20.50 & 6.0 & 111 & 0.70885 & -0.1 & 0.70905 & 0 & 15 & 1.1 \\
\hline $6 \mathrm{H}-4,120-125$ & 49.00 & 12.0 & 128 & 0.70873 & -1.2 & 0.70909 & 2 & 34 & 1.7 \\
\hline $9 \mathrm{H}-4,120-125$ & 77.80 & 23.0 & 147 & 0.70857 & -1.7 & 0.70811 & 309 & 989 & 1.2 \\
\hline $12 \mathrm{H}-4,115-125$ & 106.65 & 32.0 & 156 & 0.70852 & -1.2 & 0.70797 & 348 & 1015 & 1.1 \\
\hline $15 \mathrm{H}-4,115-125$ & 135.55 & 38.0 & 147 & 0.70834 & -1.5 & 0.70775 & 351 & 1459 & 1.3 \\
\hline $18 \mathrm{H}-4,115-125$ & 164.45 & 44.5 & 194 & 0.70822 & -1.4 & 0.70778 & 309 & 1171 & 1.2 \\
\hline $22 X-4,115-125$ & 203.15 & 55.8 & 200 & 0.70812 & -0.9 & 0.70780 & 349 & 783 & 1.3 \\
\hline $25 \times-4,115-125$ & 232.25 & 66.4 & 209 & 0.70807 & -0.9 & 0.70775 & 352 & 887 & 1.3 \\
\hline $28 X-3,115-125$ & 259.72 & 70.0 & 219 & 0.70808 & -0.4 & 0.70783 & 359 & 1102 & 1.6 \\
\hline
\end{tabular}

\footnotetext{
a Interstitial water.

belative to SMOW.

celeased by extraction with $0.1 \mathrm{~N} \mathrm{HCl}$ (ppt = parts per thousand, $\mathrm{ppm}=$ parts per million).
} 
Table 2. Interstitial water concentration data and concentration of elements in bulk sediment (extracted with $0.1 \mathrm{~N} \mathrm{HCl}$ ), Site 690 .

\begin{tabular}{|c|c|c|c|c|c|c|c|c|c|}
\hline $\begin{array}{l}\text { Core, section, } \\
\text { interval }(\mathrm{cm})\end{array}$ & $\begin{array}{l}\text { Depth } \\
\text { (mbsf) }\end{array}$ & $\begin{array}{l}\text { Age } \\
(\mathrm{Ma})\end{array}$ & $\begin{array}{l}\mathrm{Sr}^{2+} \\
(\mu \mathrm{m})\end{array}$ & $\begin{array}{l}{ }^{87} \mathrm{Sr} /{ }^{86} \mathrm{Sr} \\
\text { (I.W) }\end{array}$ & $\begin{array}{l}\delta^{18} \mathrm{O} \\
(\%)^{\mathrm{b}}\end{array}$ & $\begin{array}{l}{ }^{87} \mathrm{Sr} /{ }^{86} \mathrm{Sr} \\
(\text { A.E. })^{c}\end{array}$ & $\underset{(\mathrm{ppt})^{\mathrm{c}}}{\mathrm{Ca}}$ & $\underset{(\mathrm{ppm})^{\mathrm{c}}}{\mathrm{Sr}}$ & $\underset{\text { (ppt) }}{\mathrm{Mg}}$ \\
\hline \multicolumn{10}{|l|}{ 113-690B- } \\
\hline $2 \mathrm{H}-3,145-150$ & 6.55 & 5.0 & 99 & 0.70953 & -0.5 & 0.70940 & 1 & 18 & 1.3 \\
\hline $3 \mathrm{H}-4,120-125$ & 17.40 & 6.5 & 104 & 0.70911 & -0.5 & 0.70942 & 1 & 19 & 1.3 \\
\hline $6 \mathrm{H}-4,120-125$ & 46.50 & 17.5 & 109 & 0.70887 & -1.1 & 0.70864 & 135 & 627 & 1.7 \\
\hline $9 \mathrm{H}-4,120-125$ & 75.50 & 28.0 & 138 & 0.70880 & -1.4 & 0.70806 & 320 & 994 & 1.7 \\
\hline $12 \mathrm{H}-5,120-125$ & 106.00 & 35.0 & 127 & 0.70859 & -2.1 & 0.70774 & 376 & 1191 & 1.4 \\
\hline $16 \mathrm{H}-4,120-125$ & 143.50 & 44.0 & 147 & 0.70842 & -1.6 & 0.70779 & 310 & 839 & 1.6 \\
\hline $19 \mathrm{H}-4,115-125$ & 172.55 & 49.0 & 146 & 0.70836 & -2.1 & 0.70781 & 336 & 894 & 1.1 \\
\hline $22 \mathrm{H}-3,120-125$ & 189.40 & 53.0 & 146 & 0.70831 & -2.3 & 0.70779 & 341 & 995 & 1.4 \\
\hline $25 \mathrm{H}-4,115-125$ & 209.85 & 58.0 & 155 & 0.70828 & -2.5 & 0.70781 & 366 & 1092 & 0.9 \\
\hline \multicolumn{10}{|l|}{$113-690 \mathrm{C}-$} \\
\hline $11 X-3,115-125$ & 208.35 & & 127 & 0.70832 & -1.5 & 0.70783 & 294 & 834 & 1.7 \\
\hline $14 X-4,115-125$ & 238.85 & 65.0 & 124 & 0.70823 & -1.5 & 0.70792 & 373 & 1000 & 1.2 \\
\hline $17 X-2,115-125$ & 264.45 & 69.0 & 160 & 0.70819 & -1.5 & 0.70787 & 279 & 960 & 1.4 \\
\hline $20 X-4,115-125$ & 296.45 & 72.0 & 159 & 0.70828 & -1.7 & 0.70788 & 364 & 962 & 5.5 \\
\hline
\end{tabular}

Table 3. Interstitial water concentration data, Site 693.

\begin{tabular}{|c|c|c|c|c|c|}
\hline $\begin{array}{l}\text { Core, section, } \\
\text { interval }(\mathrm{cm})\end{array}$ & $\begin{array}{l}\text { Depth } \\
\text { (mbsf) }\end{array}$ & $\begin{array}{l}\text { Age } \\
\text { (Ma) }\end{array}$ & $\begin{array}{l}\mathrm{Sr}^{2+} \\
(\mu \mathrm{m})\end{array}$ & $\begin{array}{c}{ }^{87} \mathrm{Sr} /{ }^{86} \mathrm{Sr} \\
(\text { I.W })^{\mathrm{a}}\end{array}$ & $\begin{array}{l}\delta^{18} \mathrm{O} \\
(\% 0)^{\mathrm{b}}\end{array}$ \\
\hline \multicolumn{6}{|l|}{$113-693 \mathrm{~A}-$} \\
\hline $2 \mathrm{R}-4,145-150$ & 8.45 & 0.5 & 76 & 0.70925 & -0.5 \\
\hline $4 \mathrm{R}-3,120-125$ & 26.10 & 2.3 & 85 & 0.70911 & -0.7 \\
\hline $6 \mathrm{R}-4,120-125$ & 46.80 & 3.0 & 94 & 0.70898 & -1.1 \\
\hline $9 R-4,120-125$ & 75.80 & 4.0 & 94 & 0.70887 & -0.7 \\
\hline $12 \mathrm{R}-4,120-125$ & 104.60 & 4.5 & 85 & 0.70886 & -1.5 \\
\hline $18 \mathrm{R}-3,120-125$ & 161.10 & 5.1 & 103 & 0.70851 & -2.3 \\
\hline $25 \mathrm{R}-2,120-125$ & 227.30 & 7.0 & 124 & 0.70835 & -2.8 \\
\hline \multicolumn{6}{|l|}{ 113-693B- } \\
\hline $2 X-3,145-150$ & 238.25 & 8.0 & 135 & 0.70847 & -3.2 \\
\hline \multicolumn{6}{|l|}{$113-693 \mathrm{~A}-$} \\
\hline $28 \mathrm{R}-3,120-125$ & 257.80 & 8.5 & 127 & 0.70840 & -1.1 \\
\hline \multicolumn{6}{|l|}{ 113-693B- } \\
\hline $6 X-4,145-150$ & 273.75 & 17.0 & 136 & 0.70810 & -1.3 \\
\hline $9 \mathrm{X}-3,120-125$ & 301.00 & 21.0 & 134 & 0.70809 & -2.9 \\
\hline $12 \mathrm{X}-3,120-125$ & 330.00 & 25.0 & 154 & 0.70784 & -2.6 \\
\hline $15 X-2,140-150$ & 357.60 & 32.5 & 138 & 0.70778 & -1.2 \\
\hline $40 \mathrm{R}-2,138-142$ & 371.78 & 32.6 & 147 & 0.70773 & -1.8 \\
\hline $17 X-1,145-150$ & 375.45 & 32.7 & 173 & 0.70773 & -3.8 \\
\hline $19 X-2,140-150$ & 396.30 & 33.0 & 166 & 0.70770 & -3.1 \\
\hline \multicolumn{6}{|l|}{$113-693 \mathrm{~A}-$} \\
\hline $48 \mathrm{R}-2,120-125$ & 448.10 & 40.0 & 168 & & \\
\hline
\end{tabular}

also present the isotope composition of the acid extracted strontium. Because the ${ }^{87} \mathrm{Sr} /{ }^{86} \mathrm{Sr}$ ratios of the acid extracts from the other sites turned out to be of little value, we only carried out a limited number of isotope ratio determinations. These are listed separately in Table 8.

Present-day seawater has an ${ }^{87} \mathrm{Sr} /{ }^{86} \mathrm{Sr}$ of 0.70920 , and most of the pore waters have a value close to this in the shallowest sediment layers.

Examination of the $\delta^{18} \mathrm{O}$ values in Tables 1-7 reveals that considerable scatter is present. In some instances (e.g., Cores
Table 4. Interstitial water concentration data, Site 694.

\begin{tabular}{lrrrrr}
\hline $\begin{array}{c}\text { Core, section, } \\
\text { interval (cm) }\end{array}$ & $\begin{array}{r}\text { Depth } \\
(\mathrm{mbsf})\end{array}$ & $\begin{array}{c}\text { Age } \\
(\mathrm{Ma})\end{array}$ & $\begin{array}{c}\mathrm{Sr}^{2+} \\
(\mu \mathrm{m})\end{array}$ & $\begin{array}{c}{ }^{87} \mathrm{Sr} /{ }^{86} \mathrm{Sr} \\
(\mathrm{I} . \mathrm{W})^{\mathrm{a}}\end{array}$ & $\begin{array}{r}\delta^{18} \mathrm{O} \\
(\%)^{\mathrm{b}}\end{array}$ \\
\hline $113-694 \mathrm{~B}-$ & & & & & \\
& & & & & \\
1H-2, 145-150 & 2.95 & 0.8 & 83 & 0.70914 & -0.3 \\
$3 \mathrm{H}-2,120-125$ & 17.79 & 4.5 & 92 & 0.70919 & -0.8 \\
$22 \mathrm{X}-1,145-150$ & 151.85 & 5.4 & 93 & 0.71063 & -0.6 \\
$24 \mathrm{X}-2,120-125$ & 172.30 & 6.8 & 93 & 0.70967 & -1.1 \\
& & & & & \\
$13-694 \mathrm{C}-$ & & & & & \\
& & & & & \\
5X-1, 145-150 & 210.35 & 8.5 & 102 & 0.70998 & -1.8 \\
$14 X-4,115-125$ & 300.35 & 12.6 & 102 & 0.71017 & -2.6 \\
$19 X-2,145-150$ & 345.95 & 15.0 & 111 & 0.71025 & -1.1 \\
22X-2, 115-125 & 374.65 & & 115 & 0.70983 & -2.7 \\
\hline
\end{tabular}

${ }^{8}{ }^{87} \mathrm{Sr} /{ }^{86} \mathrm{Sr}$ ratio of interstitial water strontium.

${ }^{b}$ Relative to SMOW.

Table 5. Interstitial water concentration data, Site 695.

\begin{tabular}{crrrrr}
\hline $\begin{array}{c}\text { Core, section, } \\
\text { interval (cm) }\end{array}$ & $\begin{array}{c}\text { Depth } \\
(\mathrm{mbsf})\end{array}$ & $\begin{array}{c}\text { Age } \\
(\mathrm{Ma})\end{array}$ & $\begin{array}{r}\mathrm{Sr}^{2+} \\
(\mu \mathrm{m})\end{array}$ & $\begin{array}{r}{ }^{87} \mathrm{Sr} /{ }^{86} \mathrm{Sr} \\
(\mathrm{I} . \mathrm{W})^{\mathrm{a}}\end{array}$ & $\begin{array}{r}\delta^{18} \mathrm{O} \\
(\% 0)^{\mathrm{b}}\end{array}$ \\
\hline $113-695 \mathrm{~A}-$ & & & & & \\
& & & & & \\
$1 \mathrm{H}-3,145-150$ & 4.45 & 1.4 & 93 & 0.70919 & -1.2 \\
$3 \mathrm{H}-4,140-145$ & 18.10 & 2.8 & 89 & 0.70912 & 0.0 \\
6H-3, 120-125 & 45.40 & 3.3 & 90 & 0.70910 & -1.2 \\
$9 \mathrm{H}-2,120-125$ & 72.70 & 3.4 & 103 & 0.70897 & -0.9 \\
$12 \mathrm{H}-4,120-125$ & 94.90 & 3.6 & 99 & 0.70890 & -1.5 \\
$15 \mathrm{H}-3,120-125$ & 122.40 & 3.8 & 110 & 0.70886 & -1.3 \\
$19 \mathrm{X}-4,120-125$ & 153.00 & 4.0 & 115 & 0.70876 & -1.4 \\
$22 \mathrm{X}-3,120-125$ & 180.70 & 4.1 & 118 & 0.70857 & -1.8 \\
$25 \mathrm{X}-3,120-125$ & 200.10 & 14.2 & 120 & 0.70874 & -2.1 \\
$29 \mathrm{X}-2,115-125$ & 237.35 & 4.4 & 124 & 0.70879 & -2.1 \\
$32 \mathrm{X}-4,115-125$ & 259.75 & 4.5 & 125 & 0.70880 & -1.3 \\
$36 \mathrm{X}-2,115-125$ & 295.45 & 4.8 & 131 & 0.70879 & -1.8 \\
$39 \mathrm{X}-3,115-125$ & 316.15 & 5.1 & 127 & 0.70887 & -2.2 \\
$41 \mathrm{X}-3,140-150$ & 335.80 & 5.3 & 134 & 0.70877 & -2.3 \\
\hline & & & & & \\
\hline
\end{tabular}

${ }^{\text {a }}{ }^{87} \mathrm{Sr} /{ }^{86} \mathrm{Sr}$ ratio of interstitial water strontium.

b Relative to SMOW. 
Table 6. Interstitial water concentration data, Site 696.

\begin{tabular}{lrrrrr}
\hline $\begin{array}{c}\text { Core, section, } \\
\text { interval }(\mathrm{cm})\end{array}$ & $\begin{array}{c}\text { Depth } \\
(\mathrm{mbsf})\end{array}$ & $\begin{array}{c}\text { Age } \\
(\mathrm{Ma})\end{array}$ & $\begin{array}{c}\mathrm{Sr}^{2+} \\
(\mu \mathrm{m})\end{array}$ & $\begin{array}{c}{ }^{87} \mathrm{Sr} /{ }^{86} \mathrm{Sr} \\
(\mathrm{I} . \mathrm{W})^{\mathrm{a}}\end{array}$ & $\begin{array}{c}\delta^{18} \mathrm{O} \\
(\% 0)^{\mathrm{b}}\end{array}$ \\
\hline 113-696A- & & & & & \\
& & & & & \\
2H-3, 120-125 & 6.70 & 0.6 & 112 & 0.70897 & -0.4 \\
5H-3, 120-125 & 35.40 & 3.6 & 125 & 0.70843 & -2.2 \\
8H-4, 120-125 & 65.70 & 4.3 & 123 & & -2.7 \\
& & & & & \\
113-696B- & & & & & \\
& & & & & -2.0 \\
3R-3, 120-125 & 90.40 & 4.4 & 123 & & -3.5 \\
6R-3,120-125 & 119.40 & 4.7 & 136 & 0.70764 & -2.4 \\
20R-2, 120-125 & 233.80 & 7.0 & 171 & 0.70681 & -3.5 \\
26R-2, 120-125 & 291.70 & 8.4 & 172 & & -2.9 \\
34R-1, 120-125 & 367.50 & 10.0 & 162 & 0.70722 & -2.9 \\
53R-4, 115-125 & 554.55 & 20.0 & 168 & 0.70766 & -3.9 \\
59R-3, 115-125 & 611.05 & 31.0 & 160 & 0.70768 & -4.1 \\
62R-5, 115-125 & 641.96 & 41.0 & 167 & 0.70748 & -4.0 \\
\hline
\end{tabular}

${ }^{\text {a }}{ }^{87} \mathrm{Sr} /{ }^{86} \mathrm{Sr}$ ratio of interstitial water strontium.

${ }^{\mathrm{b}}$ Relative to SMOW.

113-696B-3R-3, 113-696B-26R-2, and 113-696B-34R-1) anomalous $\delta^{18} \mathrm{O}$ values are associated with anomalous sulfate concentrations, thus some contamination by seawater may have taken place. However, in most cases the scatter in the oxygen isotope data is not related to fluctuations in the concentration of any of the other dissolved constituents. The fluctuations are much larger than what one would expect from the reproducibility of the method $( \pm 0.2 \%)$ and are probably partly due to analytical difficulties. We believe the data represent the general trends, but do not trust them sufficiently to go into any detailed discussion.
Table 7. Interstitial water concentration data, Site 697.

\begin{tabular}{lrrrrr}
\hline $\begin{array}{c}\text { Core, section, } \\
\text { interval (cm) }\end{array}$ & $\begin{array}{c}\text { Depth } \\
(\mathrm{mbsf})\end{array}$ & $\begin{array}{c}\text { Age } \\
(\mathrm{Ma})\end{array}$ & $\begin{array}{c}\mathrm{Sr}^{2+} \\
(\mu \mathrm{m})\end{array}$ & $\begin{array}{c}{ }^{87} \mathrm{Sr} /{ }^{86} \mathrm{Sr} \\
(\mathrm{I} . \mathrm{W})^{\mathrm{a}}\end{array}$ & $\begin{array}{c}\delta^{18} \mathrm{O} \\
(\%(\%)\end{array}$ \\
\hline $113-697 \mathrm{~b}-$ & & & & & \\
& & & & & \\
1H-4, 145-150 & 5.95 & 0.1 & 89 & 0.70915 & -1.2 \\
& & & & & \\
$113-697 \mathrm{~B}-$ & & & & & \\
& & & & & \\
1H-1, 145-150 & 19.45 & 0.4 & 89 & 0.70923 & -1.4 \\
$3 \mathrm{H}-4,120-125$ & 42.80 & 0.9 & 97 & & -1.0 \\
$4 \mathrm{H}-4,115-125$ & 52.45 & 1.2 & 95 & 0.70950 & -1.6 \\
$5 \mathrm{H}-3,115-125$ & 60.65 & 1.4 & 89 & 0.70914 & -1.5 \\
$6 \mathrm{H}-2,115-125$ & 68.85 & 1.6 & 90 & 0.70913 & -1.0 \\
$7 \mathrm{H}-2,140-150$ & 78.90 & 1.9 & 93 & 0.70912 & -2.5 \\
$8 \mathrm{H}-3,140-150$ & 90.10 & 2.2 & 93 & & -3.3 \\
$9 \mathrm{H}-3,115-125$ & 99.55 & 2.3 & 93 & & -1.4 \\
$13 \mathrm{X}-3,115-125$ & 132.75 & 3.0 & 102 & 0.70911 & -0.6 \\
$16 \mathrm{X}-4,115-125$ & 163.15 & 3.4 & 106 & 0.70911 & \\
$19 \mathrm{X}-3,115-125$ & 190.75 & 3.7 & 100 & 0.70911 & -0.9 \\
$22 \mathrm{X}-2,115-125$ & 218.15 & 3.9 & 103 & 0.70911 & -1.6 \\
$26 \mathrm{X}-2,115-125$ & 256.85 & 4.1 & 101 & 0.70925 & -1.8 \\
$29 \mathrm{X}-2,115-125$ & 285.90 & 4.3 & 79 & 0.70926 & \\
$31 \mathrm{X}-2,140-150$ & 305.50 & 4.4 & 111 & 0.70936 & -2.2 \\
\hline
\end{tabular}

${ }^{87} \mathrm{Sr} /{ }^{86} \mathrm{Sr}$ ratio of interstitial water strontium.

${ }^{\mathrm{b}}$ Relative to SMOW.

\section{Sites 689 and 690}

The sediments deposited in the interval 72-14 Ma at these sites contain more than $70 \%$ carbonate (average $84 \%$ ) Here the acid extracts give an average concentration of strontium in carbonate of $1200 \mathrm{ppm}( \pm 190 \mathrm{ppm})$ and the discrepancy between

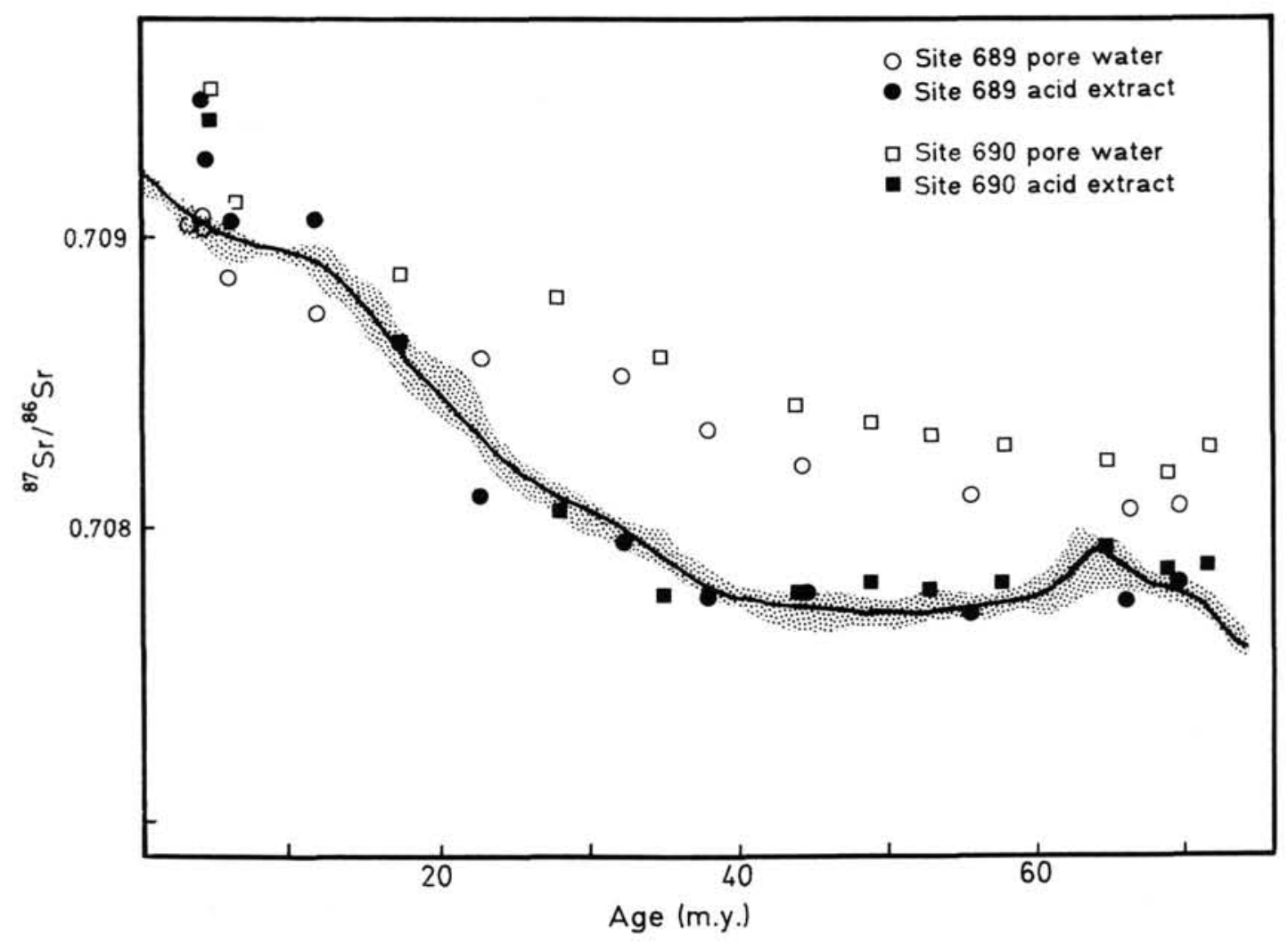

Figure $2 .{ }^{87} \mathrm{Sr} /{ }^{86} \mathrm{Sr}$-ratios of pore water strontium and acid leached strontium vs. sediment age at Sites 689 and 690 , data from Tables 1 and 2 . The shaded area represents the spread of data used to draw the time curve (solid line). 


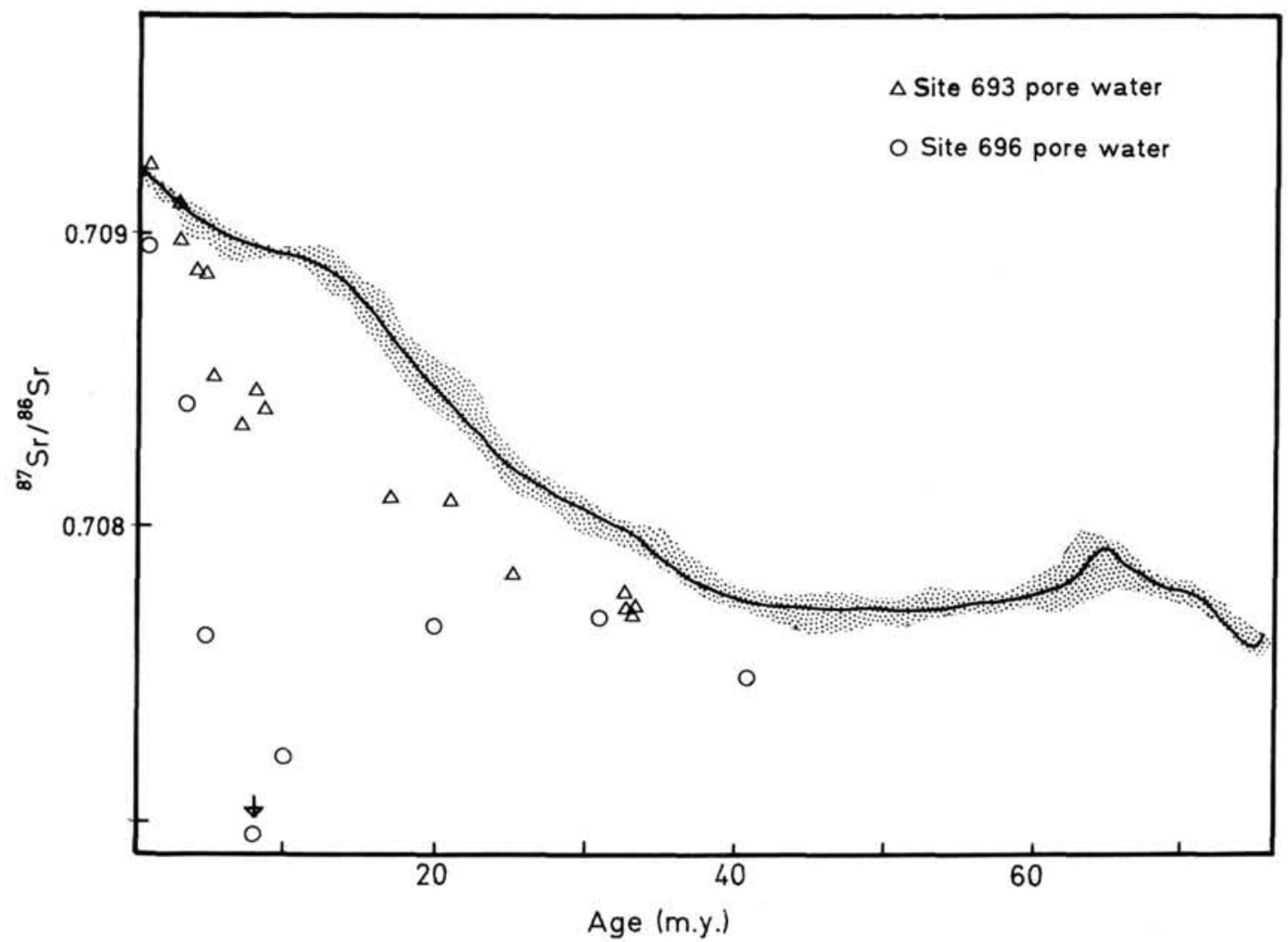

Figure $3 .{ }^{87} \mathrm{Sr} /{ }^{86} \mathrm{Sr}$-ratios of pore water strontium vs. sediment age at Sites 693 and 696 , data from Tables 3 and 6 . The shaded area represents the spread of data used to draw the time curve (solid line).

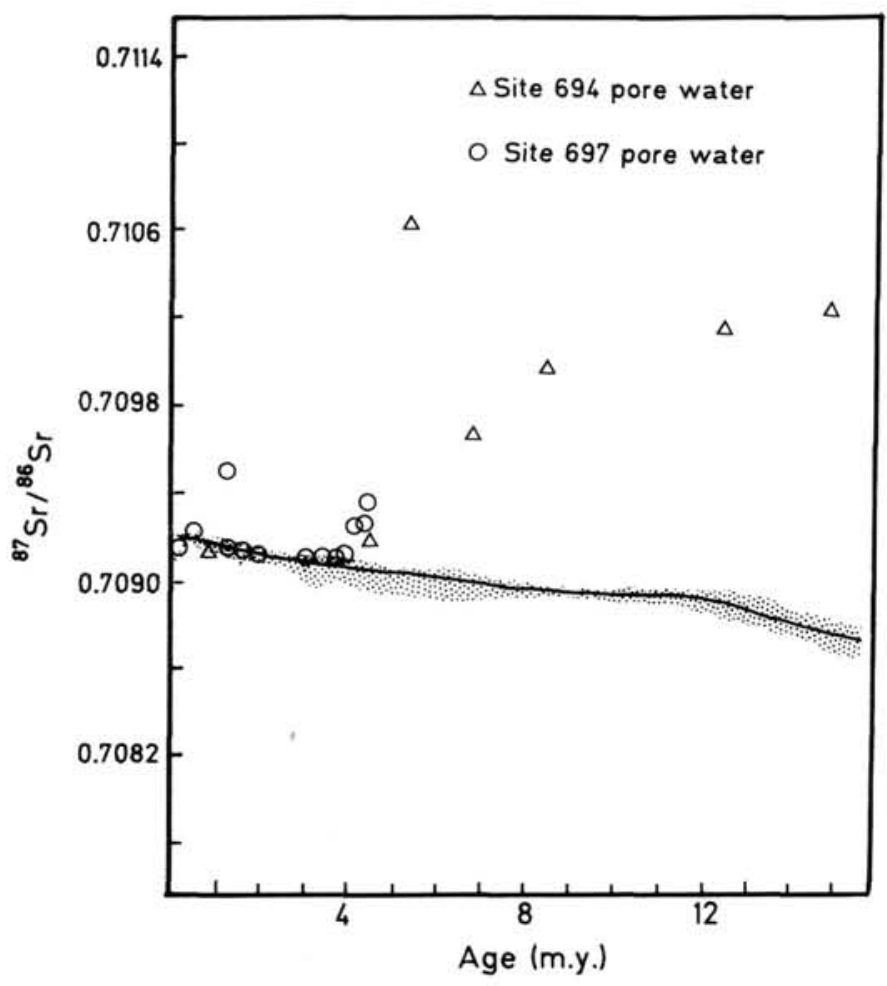

Figure $4 .{ }^{87} \mathrm{Sr} /{ }^{86} \mathrm{Sr}$-ratios of pore water strontium vs. sediment age at Sites 694 and 697, data from Tables 4 and 7. The shaded area represents the spread of data used to draw the time curve (solid line).

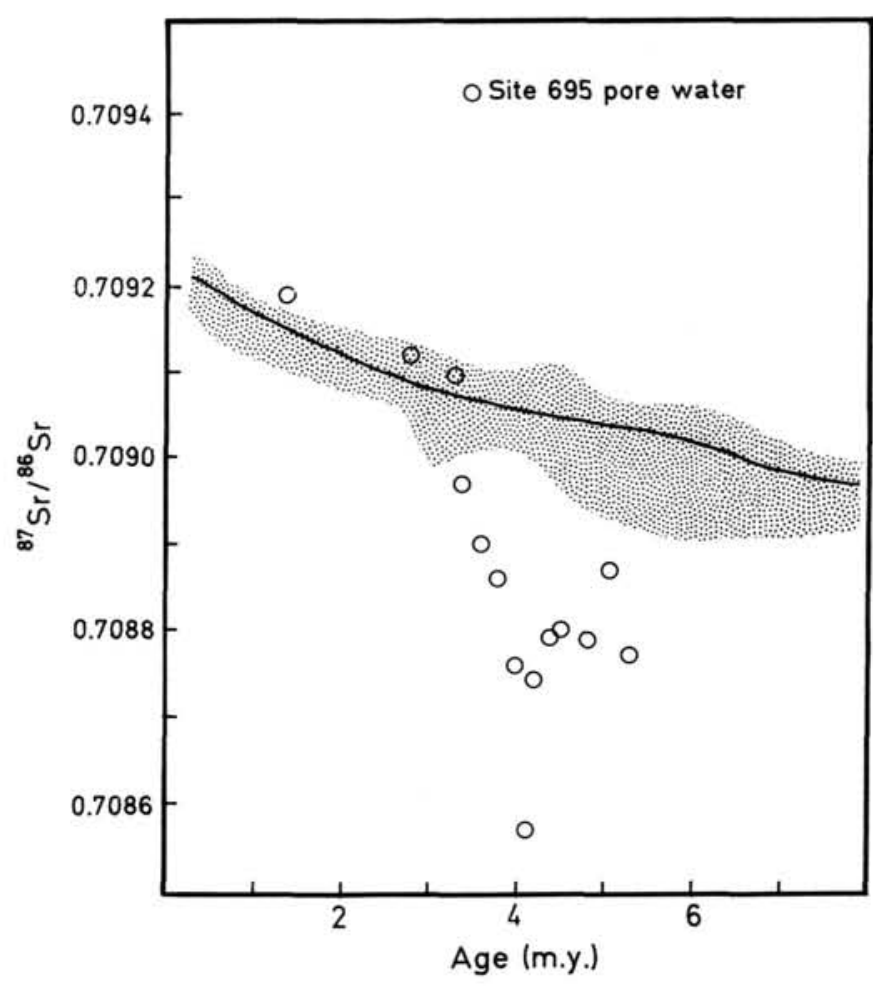

Figure $5 .{ }^{87} \mathrm{Sr} /{ }^{86} \mathrm{Sr}$-ratios of pore water strontium vs. sediment age at Site 695 , data from Table 5. The shaded area represents the spread of data used to draw the time curve (solid line). 
Table. ${ }^{8} \cdot{ }^{87} \mathrm{Sr} /{ }^{86} \mathrm{Sr}$ data on strontium extracted from bulk sediment with $0.1 \mathrm{~N} \mathrm{HCl}$. Sites 693 and 696 .

\begin{tabular}{crrr}
\hline $\begin{array}{c}\text { Core, section, } \\
\text { interval (cm) }\end{array}$ & $\begin{array}{c}\text { Depth } \\
\text { (mbsf) }\end{array}$ & $\begin{array}{c}\text { Age } \\
(\mathrm{Ma})\end{array}$ & ${ }^{87} \mathrm{Sr} /{ }^{86} \mathrm{Sr}$ \\
\hline 113-693A- & & & \\
& & & \\
2R-4, 145-150 & 8.45 & 0.8 & 0.71009 \\
6R-4, 120-125 & 46.80 & 3.0 & 0.71198 \\
12R-4, 120-125 & 104.60 & 4.5 & 0.71114 \\
25R-2, 120-125 & 227.30 & 7.0 & 0.71137 \\
28R-3, 120-125 & 257.80 & 8.5 & 0.71111 \\
& & & \\
113-693B- & & & \\
9X-3, 120-125 & 301.00 & 21.0 & 0.71102 \\
15X-2, 140-150 & 357.60 & 32.5 & 0.71147 \\
19X-2, 140-150 & 396.30 & 33.0 & 0.71102 \\
48R-2, 120-125 & 448.10 & 40.0 & 0.71238 \\
& & & \\
113-696A- & & & \\
2H-3, 120-125 & 6.70 & 0.6 & 0.70939 \\
8H-4, 120-125 & 65.70 & 4.3 & 0.70929 \\
\hline
\end{tabular}

the isotope composition of the acid liberated $\mathrm{Sr}$ and the seawater curve (Fig. 2) is less than the uncertainty in the assigned ages (Barker, Kennett, et al., 1988) In the diatomaceous shallower sections above $50 \mathrm{mbsf}$ the ${ }^{87} \mathrm{Sr} /{ }^{86} \mathrm{Sr}$ of the acid liberated strontium varies erratically between 0.7086 and 0.7094 . The concentration of dissolved strontium increases from that of seawater $(0.090$ $\mathrm{mmol} / \mathrm{L}$ ) in the shallower samples to 0.22 and $0.16 \mathrm{mmol} / \mathrm{L}$ in the deeper samples at Sites 689 and 690 respectively (Tables 1 and 2) At Site 689 the ${ }^{87} \mathrm{Sr} /{ }^{86} \mathrm{Sr}$ isotope ratio of dissolved strontium decreases smoothly from 0.70902 at 2.9 mbsf $(3.0 \mathrm{Ma})$ to 0.70808 at $259.7 \mathrm{mbsf}(70 \mathrm{Ma})$. With two exceptions, at 20.5 mbsf (6.0 Ma) and $49 \mathrm{mbsf}(\sim 12 \mathrm{Ma})$, the data plot at or above the seawater curve (Fig. 2). At Site 690 the $\mathrm{Sr}^{87} / \mathrm{Sr}^{86}$ isotope ratio of pore water strontium decreases smoothly from 0.70911 at $17.4 \mathrm{mbsf}(6.5 \mathrm{Ma})$ to 0.70819 at $264.5 \mathrm{mbsf}(69 \mathrm{Ma})$. The pore water strontium in the shallower sample $(6.6 \mathrm{mbsf}, 5.0 \mathrm{Ma}) \mathrm{ex}-$ hibits a ${ }^{87} \mathrm{Sr} /{ }^{86} \mathrm{Sr}$ ratio of 0.70953 . All data plot above the seawater curve (Fig. 2).

At Sites 693, 694, 695, 696, and 697 the sediments consist predominantly of hemipelagic deposits, very low in calcium carbonate (generally less than $0.2 \%$ ).

\section{Site 693}

The ${ }^{87} \mathrm{Sr} /{ }^{86} \mathrm{Sr}$ isotope ratio of dissolved strontium (Fig. 3) decreases smoothly from 0.70925 at $8.5 \mathrm{mbsf}(0.8 \mathrm{Ma})$ to 0.70770 at 396.3 mbsf ( $33 \mathrm{Ma}$ ) Except for the two shallowest samples all waters are less radiogenic than contemporaneous seawater. The concentration of dissolved strontium increases from $0.076 \mathrm{mmol} / \mathrm{L}$ in the shallower sample to $0.169 \mathrm{mmol} / \mathrm{L}$ at $448 \mathrm{mbsf}(40 \mathrm{Ma})$. The ${ }^{87} \mathrm{Sr} /{ }^{86} \mathrm{Sr}$ of the acid liberated strontium ranges between 0.7101 and 0.7124 .

\section{Site 694}

The ${ }^{87} \mathrm{Sr} /{ }^{86} \mathrm{Sr}$ of pore water strontium ranges between 0.70914 and 0.71063 . The two shallowest samples plot close to the seawater curve (Fig. 4) Below $172 \mathrm{mbsf}(6.8 \mathrm{Ma}){ }^{87} \mathrm{Sr} /{ }^{86} \mathrm{Sr}$ increases smoothly and reaches 0.71025 at 346 mbsf (15 Ma) before decreasing abruptly to 0.70983 at $\mathbf{3 7 4 . 7}$ mbsf (sediments at this depth could not be dated) The highest value $(0.71063)$ is observed at $151.9 \mathrm{mbsf}(5.4 \mathrm{Ma})$ The concentration of dissolved strontium increases by about $20 \%$ from the surface to the deepest sample (Table 4).

\section{Site 695}

The pore water ${ }^{87} \mathrm{Sr} /{ }^{86} \mathrm{Sr}$ varies between 0.70919 and 0.70857 . Above $45.4 \mathrm{mbsf}$ (3.3 Ma) the pore water strontium isotope ratio plots close to the seawater curve (Fig. 5) Below this, ${ }^{87} \mathrm{Sr} /{ }^{86} \mathrm{Sr}$ falls rapidly to a minimum value of 0.70857 at $180.7 \mathrm{mbsf}(4.1$ $\mathrm{Ma}$ ) below which considerable scatter is observed. The concentration of dissolved strontium increases from $0.093 \mathrm{mmol} / \mathrm{L}$ in the shallower sample to $0.135 \mathrm{mmol} / \mathrm{L}$ at $335.8 \mathrm{mbsf}(5.3 \mathrm{Ma})$.

\section{Site 696}

The ${ }^{87} \mathrm{Sr} /{ }^{86} \mathrm{Sr}$ ratio of pore water strontium decreases from 0.70897 at $6.7 \mathrm{mbsf}(0.6 \mathrm{Ma})$ to a pronounced minimum of 0.70681 at $233.8 \mathrm{mbsf}$ (7.0 Ma, Fig. 3); all of the data plot below the seawater curve. The concentration of strontium increases from $0.113 \mathrm{mmol} / \mathrm{L}$ in the shallower sample to a plateau of about $0.170 \mathrm{mmol} / \mathrm{L}$ below $233.8 \mathrm{mbsf}$ (7.0 Ma, Table 6).

\section{Site 697}

The pore water ${ }^{87} \mathrm{Sr} /{ }^{86} \mathrm{Sr}$ plots on or above the seawater curve. A maximum value of 0.70905 is observed at $52.5 \mathrm{mbsf}$ $(1.2 \mathrm{Ma})$ and the ${ }^{87} \mathrm{Sr} /{ }^{86} \mathrm{Sr}$ of the three deeper samples are also significantly higher than contemporaneous seawater (Fig. 4) The concentration of pore water strontium increases from 0.089 $\mathrm{mmol} / \mathrm{L}$ at $5.95 \mathrm{mbsf}(0.1 \mathrm{Ma})$ to $0.112 \mathrm{mmol} / \mathrm{L}$ at $305.5 \mathrm{mbsf}$ (4.4 Ma, Table 7).

\section{DISCUSSION}

The Leg 113 pore water data agree with previous work which has shown that deep sea pore waters are usually enriched in strontium relative to seawater. In the present case this may be the result of addition of strontium from various possible reservoirs: carbonates and basic and siliceous detrital material. The Leg 113 interstitial waters may be grouped into three categories according to which of these components dominate the pore water strontium.

\section{Sites 689 and 690}

At both sites there are several reservoirs of strontium. In pelagic carbonate-rich deposits strontium derived from carbonates in the host rock is generally thought to be the main control on pore water ${ }^{87} \mathrm{Sr} /{ }^{86} \mathrm{Sr}$ and strontium concentration (Baker, 1986; Baker et al., 1982; Richter and Depaolo, 1987) Interaction with basaltic basement has also been invoked (Spooner, 1976). Hawkesworth and Elderfield (1976) demonstrated that strontium released during alteration of volcanic matter may also give a strong ${ }^{87} \mathrm{Sr} /{ }^{86} \mathrm{Sr}$ signal. At Site 690 there is a significant noncarbonate component consisting of eolian material below 137 mbsf ( $42 \mathrm{Ma}$ ) most probably derived from East Antarctica. A similar component may be present at Site 689 in smaller abundance. At Site 690 two thin virtually unaltered (Schandl and Wicks, this volume) basalt flows (possibly basement) were drilled, but the composition of the underlying basement is not known. At Site 690, the sediment sequence below 275 mbsf (70 $\mathrm{Ma}$ ) consists predominantly of terrigenous material and contain $5 \%-20 \%$ carbonate.

The high ${ }^{87} \mathrm{Sr} /{ }^{86} \mathrm{Sr}$ of the pore water strontium is in marked contrast to what has been observed in other pelagic carbonaterich sequences (Richter and Depaolo, 1987; Elderfield et al., 1982) Both groups found the isotope composition of dissolved strontium to be dominated by strontium released during recrystallization of carbonates in the host rocks, thus giving pore water ${ }^{87} \mathrm{Sr} /{ }^{86} \mathrm{Sr}$ values similar to those of the corresponding carbonates. At Sites 689 and 690, however, the pore water strontium is systematically more radiogenic than the corresponding carbonates (acid extracts). 
At the carbonate-dominated Lord Howe Rise (Richter and Depaolo, 1987) and the Ontong Java Plateau (Elderfield et al., 1982) locations, the pore waters rapidly reach stable levels of 0.7 and $0.8 \mathrm{mmol} / \mathrm{L}$ strontium respectively, whereas at Sites 689 and 690 the concentrations of dissolved strontium increase almost linearly downward from the seafloor to maximum values of 0.22 and $0.16 \mathrm{mmol} / \mathrm{L}$ respectively. This difference in strontium distribution is not related to differences in the $\mathrm{Sr} / \mathrm{Ca}$ ratio of the carbonates $(0.0029$ to 0.0042 at Maud Rise, 0.0005 to 0.0015 at Ontong Java Plateau), or to different $\mathrm{Mg} / \mathrm{Ca}$ ratios (Table 1 and 2). The Maud Rise, Lord Howe Rise, and Ontong Java Plateau sediments consist predominantly of nannofossil oozes and chalks. The difference in rate of deposition is not sufficiently large to account for the observed features, thus the comparatively low concentrations of pore water strontium in Maud Rise sediments must be due to low rates of recrystallization of the carbonates.

Based on experimental studies, Baker et al. (1980) found that the most important factors governing the rate of recrystallization of natural carbonates are temperature, effective stress and the amount of noncarbonate material. At the Lord Howe Rise and the Ontong Java Plateau, the pore waters attain their high contents of strontium within the upper 100-200 m, thus the differences in rate of recrystallization do not seem to be directly related to differences in effective stress. Baker et al. (1980) found that, qualitatively, the rate of recrystallization was inversely related to the amount of silica in solution at the end of the experiments, and attributed the retarding effect of noncarbonate material to surface chemical reactions involving the formation of siliceous surface complexes. In the Maud Rise sediments, the noncarbonate fraction is on the average $10 \%$ (absolute) greater than at Lord Howe Rise and Ontong Java Plateau. The concentration of dissolved silica, however, is actually lower than in Ontong Java Plateau pore waters. It seems that the question can only be resolved by detailed surface chemical methods. At this stage we can only suggest that the comparatively low strontium concentrations are due to retarded rate of recrystallization, and that the rate terms used by Baker et al. (1982) and Richter and Depaolo (1987) to describe rate of recrystallization of carbonates are not valid for Sites 689 and 690 .

The low degree of carbonate recrystallization at Sites 689 and 690 afford excellent opportunity to assess other influences on pore water composition. Evidently strontium with a higher ${ }^{87} \mathrm{Sr} /{ }^{86} \mathrm{Sr}$ isotope ratio than contemporaneous seawater must have been supplied to these pore waters. This strontium could have been derived from the seawater above or from the basement below or been generated within the sediment column. Based on a compilation of data from the DSDP program, Elderfield and Gieskes (1982) stated that no site yet studied shows unequivocal evidence of a ${ }^{87} \mathrm{Sr} /{ }^{86} \mathrm{Sr}$ isotope signal reflecting diagenesis of terrigenous detritus. They attributed similar ${ }^{87} \mathrm{Sr} /{ }^{86} \mathrm{Sr}$ profiles to diffusive communication with the overlying seawater. However, at Sites 689 and 690 , despite the smoothly decreasing ${ }^{87} \mathrm{Sr} /{ }^{86} \mathrm{Sr}$ isotope ratio with increasing depth, the increase in total strontium is more than sufficient to cause the concentration of ${ }^{87} \mathrm{Sr}$ to increase with depth. Thus there will be a net upward flux of both ${ }^{87} \mathrm{Sr}$ and ${ }^{86} \mathrm{Sr}$. Although the isotope ratio will be modified by diffusion, there is little doubt that there is a source of $\mathrm{Sr}$ with a relatively radiogenic isotope signature, situated either in the recovered sediments or below. Although the immediately underlying rocks are of basaltic composition the nature of ultimate basement is not known, and the possibility that the strontium is diffusing upward from a siliceous basement cannot be excluded.

At Site 690 the high ${ }^{87} \mathrm{Sr} /{ }^{86} \mathrm{Sr}$ strontium may possibly come from the underlying terrigenous section. By using the tracer diffusion coefficient for strontium given by $\mathrm{Li}$ and Gregory (1974) the formation factor for nannofossil oozes (Berner, 1980), and a porosity of 0.5 (chosen as an average for the whole sequence) the distance of diffusion during $70 \mathrm{~m} . \mathrm{y}$. is more than $700 \mathrm{~m}$. Thus, there is no doubt that if there is a supply of strontium from below, then diffusion will be rapid enough for the signal to be detected throughout the 300 -m-thick sediment column. However, the fact that below 90 mbsf (about $30 \mathrm{Ma}$ ), the ${ }^{87} \mathrm{Sr} /{ }^{86} \mathrm{Sr}$ values run almost parallel to the seawater curve (Fig. 2) suggests that the system is buffered by strontium derived from carbonates in the host rocks. By assuming that the concentration of seawater strontium has remained constant over the last 70 m.y. and that the pore waters were deposited in isotopic equilibrium with ocean water and marine carbonate, and by ignoring diffusion, the relative amount of strontium released from carbonates and terrigenous material may be estimated.

The most likely source of the terrigenous material is Queen Maud Land. Igneous rocks from this area typically have ${ }^{87} \mathrm{Sr} /$ ${ }^{86} \mathrm{Sr}$ ratios of 0.728 (Barton and Copperthwaite, 1983).

In this study we recognize four reservoirs of strontium: indigenous seawater, marine carbonate minerals, and basaltic and siliceous detrital material. Because for each site we are able to eliminate one of these sources (based on geochemical considerations) and because we assume that the original pore water was deposited in isotopic equilibrium with seawater, we are left with only two variables. By making educated guesses of the ${ }^{87} \mathrm{Sr} /{ }^{86} \mathrm{Sr}$ ratios of the strontium in these reservoirs we are able to estimate the relative amount of strontium from each of them by manipulating equations 1 and 2 .

$$
\begin{gathered}
\mathrm{Sr}_{\text {to }}=\mathrm{Sr}_{\mathrm{w}}+\mathrm{Sr}_{1}+\mathrm{Sr}_{2} \\
\mathrm{R}=\left(\Sigma \mathrm{Sr}_{1} \cdot \mathrm{R}_{\mathrm{i}} / \mathrm{K}+\mathrm{R}_{\mathrm{i}}\right) /\left(\Sigma \mathrm{Sr}_{\mathrm{i}} / \mathrm{K}+\mathrm{R}_{\mathrm{i}}\right)
\end{gathered}
$$

Where $\mathrm{Sr}_{\mathrm{to}}$ is the observed total number of moles of strontium per unit volume, $\mathrm{Sr}_{w}, \mathrm{Sr}_{1}$, and $\mathrm{Sr}_{2}$ are the number of moles derived from indigeneous seawater and reservoir 1 and 2 , respectively. $R_{0}$ and $R_{i}$ are the observed ${ }^{87} \mathrm{Sr} /{ }^{86} \mathrm{Sr}$ isotope ratio and the isotope ratio of reservoir $\mathrm{i}$, respectively. The summations are carried out over all end members. $\mathrm{K}$ is a constant given by equation 3 that relates the total number of moles of strontium $\left(\mathrm{Sr}_{\mathrm{ti}}\right)$ to the ${ }^{87} \mathrm{Sr} / 8^{6} \mathrm{Sr}$ ratio $\left(\mathrm{R}_{\mathrm{i}}\right)$ and number of moles of ${ }^{86} \mathrm{Sr}$.

$$
\mathrm{Sr}_{\mathrm{ti}}=\left(\mathrm{K}+\mathrm{R}_{\mathrm{i}}\right) \cdot{ }^{86} \mathrm{Sr}_{\mathrm{i}}
$$

The figures used to estimate $\mathrm{K}$ are those recommended by the Subcommission on Geochronology of the International Union of Geological Sciences and given by Faure (1986).

Because we are mainly interested in the relative amount of strontium derived from each source we are not considering diffusion. Implicitly we assume that all reactions are taking place simultaneously.

The profiles in Figure 6 are determined by the means of equation 1 and 2 and show the contribution to pore water $\mathrm{Sr}$ of material derived from carbonate and siliceous sources. The profiles reveal two important aspects. First, that instead of showing a systematic increase with increasing depth as one would expect if strontium with a high ${ }^{87} \mathrm{Sr} /{ }^{86} \mathrm{Sr}$ isotope ratio was supplied from below, the amount of strontium derived from terrigenous material is almost constant throughout. Secondly, assuming the choice of ${ }^{87} \mathrm{Sr} /{ }^{86} \mathrm{Sr}$ isotope ratio of the terrigenous strontium to be 0.728 , it takes only around $3 \%$ of this strontium to produce the high ${ }^{87} \mathrm{Sr} /{ }^{86} \mathrm{Sr}$ ratios in the pore waters. It appears that there is an internal source of strontium with a high ${ }^{87} \mathrm{Sr} /{ }^{86} \mathrm{Sr}$ isotope ratio. Most likely this strontium is derived from the eolian terrigenous material within the sediments. The fact that one estimates about the same contribution of terrigenous strontium at the two sites, despite the much lower abundance of terrigenous material at Site 689 , indicates that the availability of radiogenic 


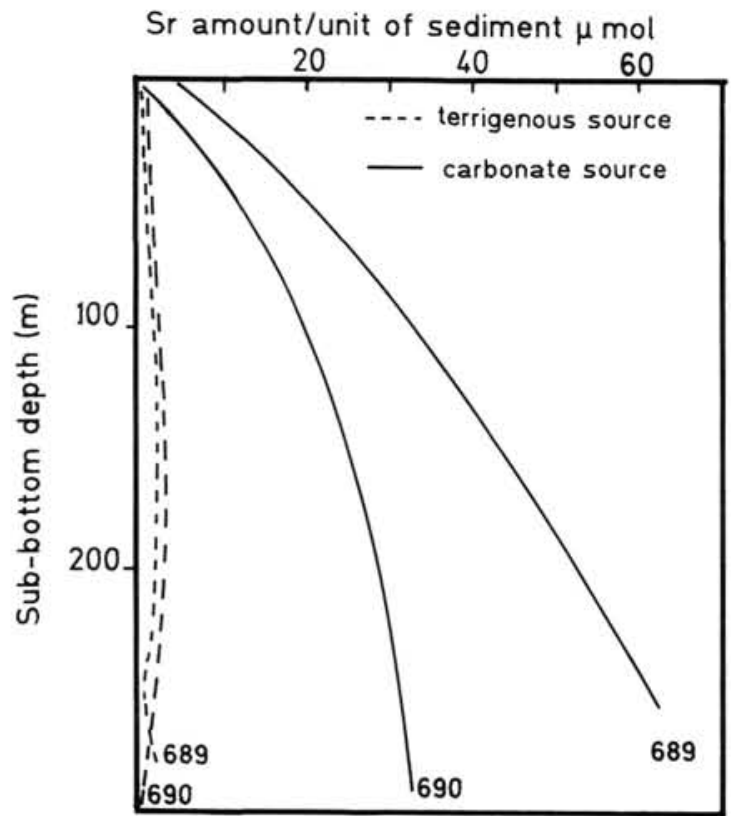

Figure 6. Amount of pore water strontium derived from terrigenous and carbonate sources at Sites 689 and 690, estimated by the means of equation 1 and 2 and by assuming a ${ }^{87} \mathrm{Sr} /{ }^{86} \mathrm{Sr}$ ratio for the terrigenous strontium of 0.728 .

strontium is not directly related to the amount of bulk eolian material.

\section{Sites 693, 695, and 696}

At Sites 693, 695, and 696 the strontium isotope signature of the pore waters plot at or below the seawater curve (Figs. 3 and 5). Low ${ }^{87} \mathrm{Sr} /{ }^{86} \mathrm{Sr}$ isotope ratios in pore waters have previously been attributed to interaction with basaltic volcanic material (Hawkesworth and Elderfield, 1976; Elderfield and Gieskes; 1982).

Egeberg et al. (this volume) show that most of the variations in major element chemistry of Leg 113 interstitial waters may be interpreted in terms of reactions involving alteration of volcanic detritus dispersed in the sediments. The irregular $\mathrm{Sr}$ isotope variations in pore waters at Sites 695 and 696 confirm that there are local sources of strontium involved. The low ${ }^{87} \mathrm{Sr} /{ }^{86} \mathrm{Sr}$ ratios of dissolved strontium suggest that much of the strontium added to the pore waters to account for the downward increase in strontium content is derived from volcanic material. This is further substantiated by the significant correlation between the $\delta^{18} \mathrm{O}$ signatures of the pore waters and the ${ }^{87} \mathrm{Sr} /{ }^{86} \mathrm{Sr}$ ratios of dissolved strontium (Fig. 7) At these sites the concentration of carbonate is very low (less than $0.1 \%$, Barker, Kennett, et al., 1988) and the contribution of carbonate strontium to the pore water may be neglected. Thus the pore water strontium may be considered as a mixture of indigenous seawater, strontium derived from altered basic volcanic material, and siliceous terrigenous material. The relative contribution of strontium from these sources may be estimated by assigning each an appropriate ${ }^{87} \mathrm{Sr} /{ }^{86} \mathrm{Sr}$ ratio. The most conservative estimate of the amount of strontium derived from basic volcanics is obtained assigning them the lowest reasonable ${ }^{87} \mathrm{Sr} /{ }^{86} \mathrm{Sr}$ ratio for local volcanic matter $(0.703)$ For strontium derived from terrigenous material we may use the same ${ }^{87} \mathrm{Sr} /{ }^{86} \mathrm{Sr}$ ratio as above $(0.728)$. Figure 8 shows the amount of strontium the pore water in each unit of pore water could have acquired from the two sources (assuming constant porosity of 0.5 ) The amount of strontium supplied from terrigenous material is about the same at all sites $(0-5 \mu \mathrm{mol})$, whereas the

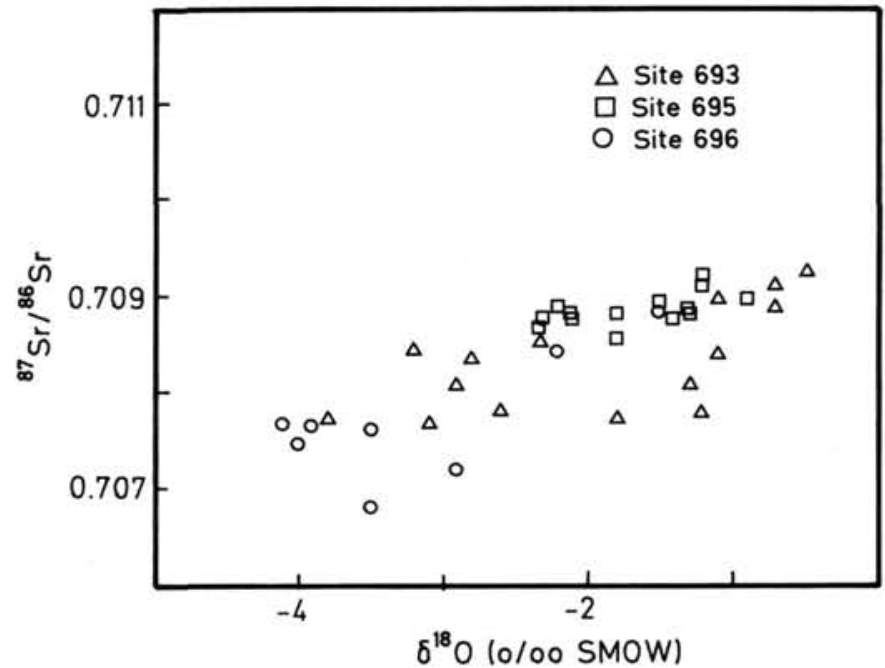

Figure $7 .{ }^{87} \mathrm{Sr} /{ }^{86} \mathrm{Sr}$-ratios of pore water strontium vs. oxygen isotope distribution at Sites 693, 695, and 696, data from Tables 3, 5, and 6.

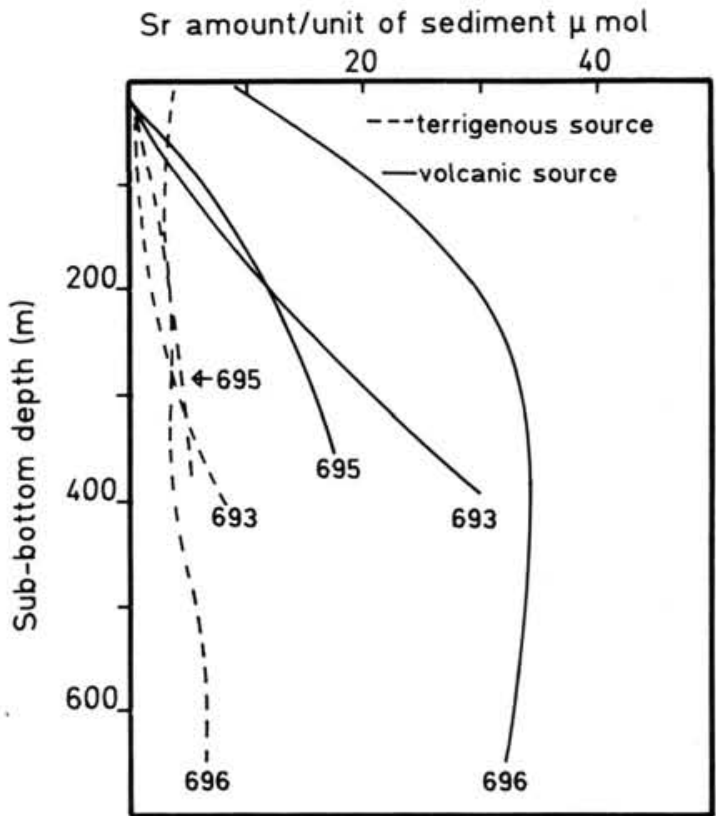

Figure 8. Amount of pore water strontium derived from terrigenous and basic volcanic sources at Sites 693,695 , and 696 , estimated by the means of equation 1 and 2 and by assuming a ${ }^{87} \mathrm{Sr} /{ }^{86} \mathrm{Sr}$ ratio of terrigenous strontium of 0.728 for the terrigenous strontium and 0.703 for the volcanogenic strontium.

amount derived from basic volcanic matter is considerably higher at Site 696 compared to the other sites. The pronounced drop in the ${ }^{87} \mathrm{Sr} /{ }^{86} \mathrm{Sr}$ ratio at Sites 695 and 696 (at $180.7 \mathrm{mbsf}(4.1 \mathrm{Ma})$ and $233.8 \mathrm{mbsf}(7.0 \mathrm{Ma})$ respectively) is due to high concentrations of volcanically derived strontium relative to that derived from terrigenous material.

An independent test of the assumptions involved in the calculations may be obtained by comparing the relation between the $\delta^{18} \mathrm{O}$ signature of the pore waters and the estimated amount of volcanically derived strontium with that predicted by alteration of volcanic material as proposed by Egeberg et al. (this volume) The curve in Figure 9 is constructed using an average concentra- 


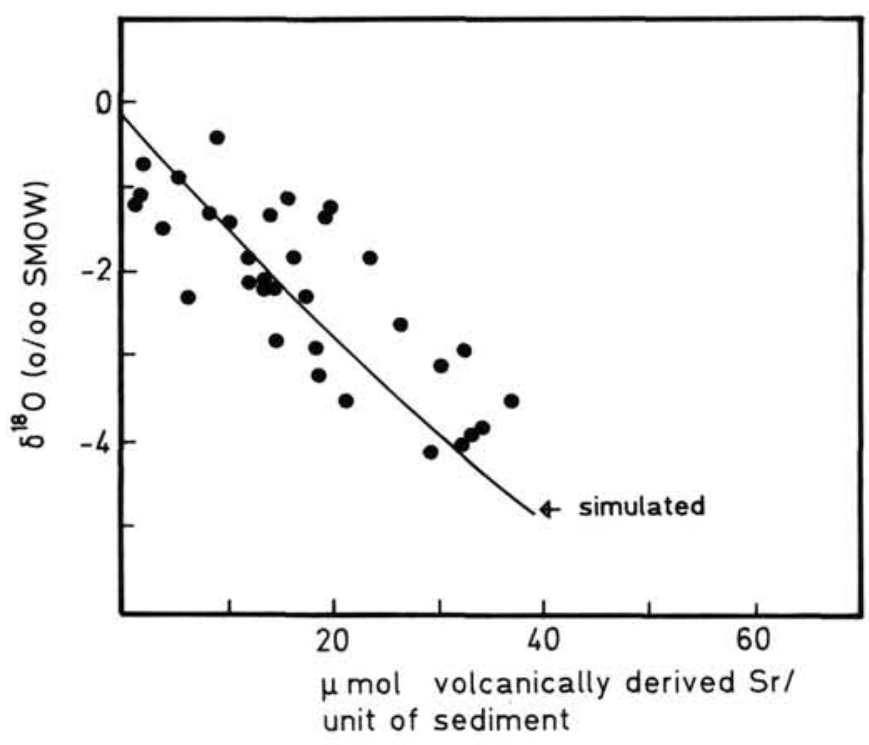

Figure 9. Oxygen isotope distribution (data from Tables 3, 5, and 6) vs. amount of strontium derived from alteration of basic volcanic material (estimated by the means of equation 1 and 2) The solid line is established by the means of equation 5 and 6 in Egeberg et al. (this volume) and by assuming a concentration of strontium in volcanic glass of 100 ppm.

tion of strontium in basaltic material of $100 \mathrm{ppm}$ (Spooner, 1976) Inspection of Figure 9 reveals a good simulation of the overall trend. Much of the scatter may be attributed to compositional variations in the reacting material.

\section{Sites 694 and 697}

The pore water strontium at Site 694 probably has the most radiogenic ${ }^{87} \mathrm{Sr} /{ }^{86} \mathrm{Sr}$ values yet observed at DSDP or ODP sites. The average ${ }^{87} \mathrm{Sr} /{ }^{86} \mathrm{Sr}$ ratio of dissolved strontium in Site 694 pore water is 0.70986 . Ratios $\left({ }^{87} \mathrm{Sr} /{ }^{86} \mathrm{Sr}\right)$ greater than that of present-day seawater $(0.70920)$ are rare in DSDP/ODP pore waters.

As with the other Leg 113 sites, the variations in major element chemistry at Sites 694 and 697 may be interpreted in terms of alteration of volcanic debris dispersed in the sediments (Egeberg et al., this volume) Evidently the effect of volcanically derived strontium is completely masked by strontium derived from terrigenous material. At Site 697 unusually high $87 \mathrm{Sr} / 87 \mathrm{Sr}$ isotope ratios are confined to the deeper samples.

The poor core recovery at Site 694 was attributed to the presence of coarse sands, and large parts of the recovered sequence consist of turbidites with abundant lithic fragments. Site 694 may be the first DSDP/ODP site where the presence of strontium released from terrigenous material is unequivocal in the pore water.

At Site 694 the content of sedimentary carbonate is so low (less than $0.1 \%$ ) that the only significant sources of strontium in the pore waters are indigenous seawater, terrigenous material, and altered basic volcanic detritus. The profiles in Figure 10 are determined using the same input parameters as at Sites 693 , 695 , and 696 . Comparison with Figure 8 shows that the high ${ }^{87} \mathrm{Sr} /{ }^{86} \mathrm{Sr}$ isotope ratios at Site 694 are not caused by excessively large amounts of terrigeneously derived strontium, but rather by the comparatively low amounts of volcanically derived strontium. Thus the radiogenic input from the terrigenous material is not fully compensated by a nonradiogenic input from basic volcanic material. Similar calculations may be carried out for Site 697.

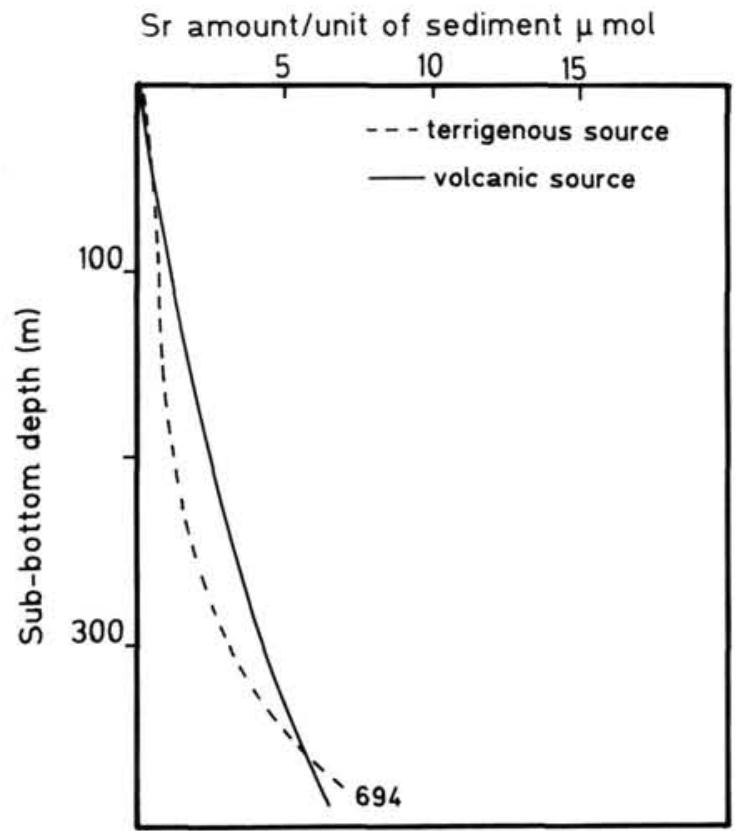

Figure 10. Amount of dissolved strontium derived from terrigenous and basic volcanic sources at Site 694, estimated by the means of equation 1 and 2 and by assuming a ${ }^{87} \mathrm{Sr} /{ }^{86} \mathrm{Sr}$ ratio of terrigenous strontium of 0.728 and 0.703 for the volcanogenic strontium.

\section{CONCLUSIONS}

The strontium concentration and the distribution of ${ }^{87} \mathrm{Sr} /$ ${ }^{86} \mathrm{Sr}$ ratios in Leg 113 interstitial waters may be interpreted in terms of mixing of strontium from four reservoirs, indigenous seawater, carbonates, altered basic volcanics, and siliceous terrigenous detrital material. The ${ }^{87} \mathrm{Sr} /{ }^{86} \mathrm{Sr}$ ratio of the pore water is determined by the magnitude of the end member isotope ratio and its reactivity, rather than by the size of the reservoir. Thus, at the carbonate-rich sites (689 and 690) the amount of strontium held in carbonates is more than 50 times the size of that held in the terrigenous material, yet the ${ }^{87} \mathrm{Sr} /{ }^{86} \mathrm{Sr}$ ratio of the interstitial waters is dominated by strontium released from terrigenous material. This indicates an almost complete lack of recrystallization of carbonates. At the hemipelagic sites the relative size of the terrigenous and basic volcanic strontium reservoir is roughly $20: 1$, yet at Sites 693,695 , and 696 , the strontium isotope composition of dissolved strontium is dominated by strontium from the reactive volcanic reservoir. Only at Site 694 and in the deepest samples from Site 697 does strontium from siliceous terrigenous material dominate. This is the result of a low contribution from the other reservoirs due to low concentrations of carbonate and limited alteration of basic volcanic material.

\section{ACKNOWLEDGMENTS}

Financial support from the Norwegian Research Council for Sciences and the Humanities and from the Norwegian Academy of Science and Letters is gratefully acknowledged.

\section{REFERENCES}

Baker, P. A., 1986. Pore water chemistry of carbonate-rich sediments, Lord Howe Rise, Southwest Pacific Ocean. In Kennett, J. P., von der Borch, C. C., Baker, P. A., et al., Init. Repts. DSDP, 90: Washington (U.S. Govt. Printing Office) 1249-1260.

Baker, P. A., Kastner, M., Byerlee, J. D., and Lockner, D. A., 1980. Pressure solution and hydrothermal recrystallization of carbonate sediments-an experimental study. Mar. Geol. 38:185-203. 
Baker, P. A., Gieskes, J. M., and Elderfield, H., 1982. Diagenesis of carbonates in deep-sea sediments-evidence from $\mathrm{Sr} / \mathrm{Ca}$ ratios and interstitial dissolved $\mathrm{Sr}^{2+}$ data. J. Sediment. Petrol., 52:71-82.

Barker, P. F., Kennett, J. P., et al., 1988. Proc. ODP, Init. Repts., 113: College Station, TX (Ocean Drilling Program)

Barton, J. M., and Copperthwaite, Y. E., 1983. Sr-isotope studies of some intrusive rocks in the Ahlmann Ridge and Annandagstoppane, Western Queen Maud Land, Antarctica. In Oliver, R. L., James, P. R., and Jago, J. B., Antarctic Earth Science: Cambridge (Cambridge Univ. Press), 59-62.

Berner, R. A., 1980. Early Diagenesis, a theoretical approach: Princeton (Princeton Univ. Press).

DePaolo, D., 1986. Detailed record of the Neogene Sr isotopic evolution of seawater from DSDP Site 590B, Geology, 14:103-106.

DePaolo D., and Ingram B., 1985. High resolution stratigraphy with Srisotopes. Science, 277:187-191.

Elderfield, H., Gieskes, J. M., Baker, P. A., Oldfield, R. K., Hawkesworth, C. J., and Miller, R., $1982 .{ }^{87} \mathrm{Sr} /{ }^{86} \mathrm{Sr}$ and ${ }^{18} \mathrm{O} /{ }^{16} 0$ ratios, in terstitial water chemistry, and diagenesis in deep-sea carbonate sediments of the Ontong Java Plateau. Geochim. Cosmochim. Acta., 46:2259-2268.

Elderfield, H., and Gieskes, J. M., 1982. Sr isotopes in interstitial waters of marine sediments from Deep Sea Drilling Project cores. $\mathrm{Na}$ ture, 300:493-497.

Faure, G., 1986. Principles of isotope geology: New York (Wiley).

Gieskes, J. M., 1973. Interstitial water studies, Leg 15-Alkalinity, pH, $\mathrm{Mg}, \mathrm{Ca}, \mathrm{Si}, \mathrm{PO}_{4}$, and $\mathrm{NH}_{4}$. In Heezen, B. C., MacGregor, I. D., Foreman, H. P., et al., Init. Repts. DSDP, 20: Washington (U.S. Govt. Printing Office), 813-829.

Gieskes, J. M., and Lawrence, J. R., 1976. Interstitial water studies, Leg 35. In Hollister, C. D., Craddock, C., Bogdanov, Y. A., et al., Init. Repts. DSDP, 35: Washington (U.S. Govt. Printing Office) 407-424.

Hawkesworth, C. J., and Elderfield, H., 1976. The strontium isotope composition of interstitial waters from Site 245 and 336, DSDP. Earth and Planet. Sci. Lett. 40:423-432.
Hess, J., Bender, M. L., and Schillinger, J. -G., 1986. Evolution of the ratio of strontium-87 to strontium-86 in seawater from Cretaceous to present. Science, 231:979-984.

Koepnick, R. B., Burke, W. H., Denison, R. E., Hetherington, E. A., Nelson, J. B., and Otto, L. E., 1985. Construction of the seawater ${ }^{87} \mathrm{Sr} /{ }^{88} \mathrm{Sr}$ curve for the Cenozoic and Cretaceous: Supporting data. Chem. Geol., 58:55-81.

Li, Y., and Gregory, S., 1974. Diffusion of ions in seawater and in deep sea sediments. Geochim. Cosmochim. Acta, 38:703-714.

Manheim, F. T., 1976. Interstitial waters of marine sediments. In Riley, J. P., and Chester, R. (Eds.), Chemical Oceanography (Vol.6): London (Academic Press), 115-186.

Manheim, F. T., and Sayles, F. L., 1974. Composition and origin of interstitial waters of marine sediments based on deep sea drill cores. In Goldberg, E. D. (Ed.), The Sea (Vol. 5): New York (Wiley-Interscience), 527-568.

McDuff, E., 1981. Major cation gradients in DSDP interstitial waters: the role of diffusive exchange between sea water and upper oceanic crust. Geochim. Cosmochim. Acta, 45:1705-1713.

Palmer, M. R., and Elderfield, H., 1985. Sr isotope composition of sea water over the past 75 Myr. Nature, 314:167-174.

Perry, E. A., Gieskes, J. M., and Lawrence, J. R., 1976. Mg, Ca, and $\mathrm{O}^{18} / \mathrm{O}^{16}$ exchange in the sediment-pore water system, Hole 149 , DSDP. Geochim. Cosmochim. Acta, 40:413-423.

Richter, F. M., and Depaolo, D. J., 1987. Numerical models for diagenesis and the Neogene $\mathrm{Sr}$ isotopic evolution of sea water from DSDP Site 590B. Earth Planet. Sci. Lett., 83:27-38.

Spooner, E.T.C., 1976. The strontium isotope composition of sea water, and sea water-oceanic crust interaction. Earth Planet. Sci. Lett,. 31: $167-174$.

Wollast, R., 1974. The silica problem. In Goldberg, E. D. (Ed.), The Sea (Vol. 5): London (Wiley-Interscience), 359-392.

Date of initial receipt: 12 January 1988

Date of acceptance: 17 November 1988

Ms 113B-170 